\title{
Reflets
}

Revue ontaroise d'intervention sociale et communautaire

\section{Les sourds-aveugles : le don au-delà de la privation}

\section{Suzanne Fortin}

Volume 4, numéro 2, automne 1998

Personnes vivant avec une incapacité

URI : https://id.erudit.org/iderudit/026231ar

DOI : https://doi.org/10.7202/026231ar

Aller au sommaire du numéro

Éditeur(s)

Reflets : Revue ontaroise d'intervention sociale et communautaire

ISSN

1203-4576 (imprimé)

1712-8498 (numérique)

Découvrir la revue

Citer ce document

Fortin, S. (1998). Les sourds-aveugles : le don au-delà de la privation. Reflets, 4(2), 179-183. https://doi.org/10.7202/026231ar

Tous droits réservés (C) Reflets : Revue ontaroise d'intervention sociale et communautaire, 1998
Ce document est protégé par la loi sur le droit d'auteur. L'utilisation des services d'Érudit (y compris la reproduction) est assujettie à sa politique d'utilisation que vous pouvez consulter en ligne.

https://apropos.erudit.org/fr/usagers/politique-dutilisation/ 


\section{Les sourds-aveugles : le don au- delà de la privation}

Suzanne Fortin, Sudbury

Ma carrière comme professeure tire à sa fin puisqu'il me reste à peine plus de trois ans d'enseignement. Mais je donnerais n'importe quoi pour exercer ma présente profession et continuer à travailler avec les enfants sourds-aveugles. Ils ont enrichi ma vie au-delà de leur compréhension.

Je travaille présentement, à titre de responsable de la gestion de ce programme, au Centre de surdicécité de l'école NotreDame du Rosaire à BlezardValley en Ontario. Cette classe existe depuis trois ans. Six enfants ayant des incapacités visuelles et auditives et des besoins complexes la composent. Il y a aussi six interprètes ou tutrices qui sont les yeux et les oreilles de ces enfants, une infirmière pour répondre à leurs besoins médicaux (tubage, thérapie des poumons, administration des médicaments, etc.) et moi. Un professeur du Centre Jules Léger d'Ottawa, qui agit comme personne ressource, continue notre formation en surdicécité durant l'année.

Quand j'ai commencé à travailler avec les enfants atteints de surdicécité, l'exemple qui me venait à l'esprit était celui d'Helen Keller, cette personne surdouée dont les accomplissements, avec l'aide de son professeur Anne Sullivan, ont émerveillé le monde entier. Mais, en réalité, les enfants sourds-aveugles tombent rarement dans cette catégorie.

Il est important de préciser que l'enfant sourd-aveugle n'est pas un enfant sourd privé de la vue, ni un enfant aveugle qui n'entend pas, ni un enfant retardé atteint de troubles visuels et 
auditifs. Ces caractéristiques rendent compte bien imparfaitement de ce qu'est l'enfant sourd-aveugle aux yeux du Comité national de l'éducation des personnes sourdes-aveugles (National Committee on the Education of the Deaf-Blind) qui en donne la définition suivante :

L'enfant sourd-aveugle est un enfant dont la combinaison des troubles visuels et auditifs résulte en une privation plurisensorielle qui fait que les méthodes traditionnelles d'éducation sur lesquelles on se base pour répondre aux besoins des enfants aveugles, sourds ou arriérés sont inadéquates.

Cette privation plurisensorielle se traduit habituellement par de nombreux problèmes dans les domaines suivants: sommeil, alimentation, habillage, discipline, activité motrice, apprentissage, expériences de vie, humeur, médical, etc. Pourtant, le problème le plus important demeure celui de la communication.

Pour pouvoir communiquer avec un enfant, il faut d'abord établir un lien affectif. Sans cette relation, la communication est impossible. Une fois les liens affectifs établis, la méthode la plus efficace pour enseigner aux enfants sourds-aveugles consiste à adopter la séquence de communication suivante: gestes, indicateurs concrets, expressions faciales, ton de la voix, langage des signes, langue signée adaptée, langage parlé, écriture, images. Pour l'enfant, apprendre à communiquer peut être un processus très long. Mais pour que l'enfant reçoive le message intact, les signes sont généralement effectués sur son corps par l'interprète ou tutrice et, ensuite, coactivement avec les mains de l'enfant. Chaque signe est adapté à son niveau de fonctionnement visuel et moteur.

L'interprète ou tutrice doit faire preuve d'une grande patience. Elle doit donner le temps à l'enfant de réagir à ce qu'elle dit. Elle doit aussi être attentive à toutes ses réactions. En raison de leurs surdicécités et de leurs limites physiques, puisqu'ils sont tous en fauteuil roulant, les enfants mettront plus de temps à assimiler l'information, à la traiter et à y réagir. Au début, certaines expressions de l'enfant passent souvent inaperçues : un signe clair, un sourire, un mouvement du doigt. Mais l'interprète ou tutrice 
prend le temps de faire tout avec l'enfant et non pas, pour l'enfant. Elle s'applique à faire les signes et encourage l'enfant à employer sa vision et son audition résiduelles. Elle prend le temps de le laisser s'exprimer et choisit des expériences ou des exercices où il aura du succès. Entre l'interprète ou tutrice et l'enfant s'établit une relation extraordinaire où règne l'amour. L'intervention est la clé, bien qu'elle demande beaucoup de temps et d'effort pour les personnes qui travaillent auprès de ces enfants. C'est une tâche sans fin, épuisante au plan émotif et physique.

Chacune des personnes (personnes spécialisées, parents, interprète, etc.) se doit d'établir un signal de communication approprié lorsqu'on approche l'enfant. Par exemple, je touche son oreille gauche et je prends sa main pour qu'il touche la mienne si possible. Ce geste lui permet d'anticiper l'activité à venir. Or, il faut établir ce genre de routines élémentaires et les suivre le plus rigoureusement possible. En somme, il faut l'avertir que l'activité commence en lui donnant un indicateur concret (éponge pour le bain, cuillère pour manger, etc.), lui donner un signe pour continuer l'activité et enfin, lui donner un autre signe pour lui signifier la fin de l'activité. Il faut faire le maximum d'efforts pour l'inciter à participer, à choisir et à employer sa vision et son audition résiduelles. L'interprète communique avec l'enfant pour indiquer l'activité, pendant et après l'activité. Il est primordial de faire l'activité avec l'enfant et non de faire l'activité pour l'enfant. Qu'il s'agisse d'atteindre un mobile qui pend au-dessus de lui, de ramasser un bloc, de pousser la table de sa chaise roulante, d'enlever son bas, toutes ces activités sont des problèmes que l'enfant apprend à résoudre au fur et à mesure qu'il grandit. Il ne faut pas lui donner la solution, mais l'aider à résoudre le problème.

Quand j'observe l'enfant assis sur les genoux de l'interprète et qu'il commence à ressentir un je-ne-sais-quoi, je peux m'imaginer le chemin de l'interaction qu'il lui restera à parcourir. Ce chemin suit généralement les étapes suivantes: l'enfant résiste à l'interaction, puis la tolère. Ensuite, l'enfant coopère passivement, puis montre qu'il apprécie l'interaction. Cette phase est suivie de celle où l'enfant répond en coopérant et par celle où il initie l'interaction. Finalement, les deux derniers stades sont ceux où 
l'enfant imite l'action et fait l'action. Ce chemin parcouru, on peut maintenant établir une programmation en fonction de ses capacités personnelles. Certains facteurs, tels les ressources communautaires, le soutien des agences, les attentes de la famille et le développement continu, influencent ce processus, mais nous n'en discuterons pas ici.

À l'école, nous travaillons en équipe avec la famille. Les parents sont une grande source d'information, de soutien, d'encouragement. Ils ont à coeur le mieux-être de leur enfant. Tous les parents de ces enfants sont exceptionnels. Ce sont aussi mes amis.J'ai toujours été touchée par leur humanité,leur ténacité, leur compassion et leur engagement dans la programmation individuelle de leur enfant. Tous les parents se connaissent et sont, l'un pour l'autre, une source d'entraide.

Les parents, les interprètes ou tutrices et surtout les enfants ne sont pas étrangers au fait que j'ai reçu, cette année, le prix prestigieux d'enseignement de TFO/TVO. Cet honneur m'a émue et je leur en suis très reconnaissante. Ce prix vient souligner la réalisation de deux projets. Le premier, qui a vu le jour grâce à une donation de $25000 \$$ de la Fondation McDonald, consiste en l'ajout, à notre école, d'une salle équipée de matériels visuels et auditifs spécialisés qui procurent aux enfants un environnement sensoriel. Cette salle porte le nom de Snoezelen et tout enfant handicapé de la région de Sudbury peut l'utiliser. C'est un peu comme leur salle de télévision. Le deuxième projet me tient beaucoup à coeur. Il s'agit de la création du premier centre francophone pour adultes, fragiles au plan médical et ayant des besoins complexes. Ce centre se nomme Le Centre Martin Asselin. Ce projet a débuté en novembre 1997 grâce à l'appui de nombreux partenaires: Conseil de district du Nouvel Ontario, Ministère des services sociaux et communautaires, Association de la Vallée pour l'intégration communautaire, Association pour les personnes ayant un handicap intellectuel du district de Sudbury. Je leur témoigne ici toute ma gratitude. Ce projet, bien qu'étant initié, est toujours à la recherche de fonds. À cet effet, les parents de ces jeunes adultes ont formé leur propre association à but non lucratif, dont je suis membre, pour recevoir les dons du public. Si cela 
vous intéresse, vous pouvez envoyer vos dons à l'adresse suivante: Destinée $21^{+}$/ Destiny $21^{+}, 2450$ Valleyview Road, Val Caron, Ontario. P3N 1R2.

J'aimerais vous laisser sur cette réflexion. Lorsque l'on regarde au-delà des limites physiques d'un enfant pour reconnaitre son potentiel intérieur, on lui donne la possibilité d'apprendre, de communiquer, de grandir et d'accomplir ce que d'aucuns estiment impossible. De cela, je suis convaincue. Pour chaque chose qu'un enfant n'a pas, il a toujours quelque chose d'autre à offrir. Il nous suffit d'ouvrir les yeux et les oreilles à ce qu'il a à donner. 